\title{
Effects on Anticipatory Postural Adjustments by Repetition of the Tiptoe Movement
}

\author{
Hiroshi SAITO, RPT, MS ${ }^{1)}$, TADAYOSHI ASAKA, RPT, PhD $^{1)}$, JUNKO FUKUSHIMA, MD ${ }^{1)}$, \\ NAOKI TAKEDA, $\mathrm{MD}^{1)}$ \\ 1) Department of Health Sciences, Hokkaido University, School of Medicine: N12 W5 \\ Kita-Ku, Sapporo City, Hokkaido 060-0812, Japan. TEL \& FAX +81 11-706-3391
}

J. Phys. Ther. Sci.

19: 83-89, 2007

\begin{abstract}
The purpose of this study was to investigate the influence of repetition on anticipatory postural adjustments between two tiptoe movement tasks. Ten healthy young male $(22.5 \pm 2.6$ years old $)$ participated in this study. They stood on tiptoe from standing position as quickly as possible after an auditory stimulus. Single and repetitive tasks as the tiptoe movement task were performed. Electromyogram during the tiptoe movement was measured in the gastrocnemius (Gas), tibialis anterior (TA), biceps femoris (BF), rectus femoris (RF), erector spinae (ES) and rectus abdominus (RA). The characteristics of the initial tiptoe movement during each task were established by analysis of kinematics data. Onsets of each muscle activity, integrated electromyography (EMG) and center of pressure (COP) displacement prior to the onset of Gas were compared between the single and repetitive tasks. The onset of TA and RF in the repetitive task was earlier than that of the single task, and integrated EMG per unit time and COP displacement also changed. These results suggest that the central nervous system may transmit different motor commands to postural muscles during single and repetitive tasks, and anticipatory postural adjustment might be modulated by the repetition of task.
\end{abstract}

Key words: Anticipatory postural adjustments (APA), Muscle activity, Center of pressure (COP)

(This article was submitted Aug. 7, 2006, and was accepted Oct. 17, 2006)

\section{INTRODUCTION}

When voluntary movements are performed ${ }^{1-8)}$ or predictable perturbation is added to the body ${ }^{9-12)}$, anticipatory postural adjustment (APA) is observed as the activation of postural muscles and/or the displacement of the center of pressure (COP) in the foot. APA is also characterized as being under the control of the central nervous system (CNS) as a feedforward mechanism, and by the earlier activation of postural muscles than the focal muscle ${ }^{13)}$. It has been supposed that the earlier postural muscle activities provide the anterior force necessary to initiate movement in tasks associated with a changing base of support (BOS), like gait initiation $^{14)}$.

Pellec et al. have reported comparison of the APA responses during single and repetitive jumping tasks initiated from the same standing posture ${ }^{15}$. They found significant differences in the activation time of the postural muscles and in the distance of the antero-posterior displacement of the COP between both jumping tasks. These suggested that even if the initial movements in both jumping tasks were similar, different APA programs might still be used in each task. That is, the CNS may modulate the duration from the onset of the postural muscle activity to the onset of the focal muscle activity (APA phase) according to the complexity of the task. However, to determine whether both jumping movements were initiated from the same movements, they measured the jump height only. They never observed the posture during jump initiation or during the jumping period, and they did 
not measure the range of motion in the joints of the lower extremities. Therefore, the methodology of their study is questionable.

Among the research associated with standing or walking, there are other studies in which different APA responses were observed in different tasks initiated from identical movements. For instance, in a study on reaching movement in standing, the duration of the anticipatory postural muscles activity increased, as the pointing target size got smaller. Therefore, the study concluded that the complexity of the task might affect APA ${ }^{16)}$. Another study examined the relationship between APA responses in the first step and the steady-state velocity in gait. It was reported that APA responses in gait initiation affected not only the first step, but also the resulting steady-state gait velocity ${ }^{17}$.

Based on these studies, we considered that the APA in the movement tasks associated with changing BOS serves not only to generate the anterior force necessary for initiation of focal movement but also to execute the optimal performance of the tasks. In other words, if APA in the tasks associated with changing BOS did more than simply generate the anterior force for initial movement, then we would expect it to change according to the complexity of the task, even though the tasks were initiated from the same movements.

The purpose of this study was to examine exactly the effects on APA of repetitive movement in tasks with changing BOS. Therefore two types of tiptoe movement task that could produce highly reproducible initial movements were used in this study. These involved movement from static standing to the tiptoe position. The APA observed in the single tiptoe task was compared to that observed in the repetitive tiptoe task where the movement was repeated three times. Both tasks were initiated from the same movement. Since our daily activities involve both single and repetitive movements, it is important to understand how the postural muscles are controlled during our daily movements.

\section{METHODS}

\section{Subjects}

The experiments were performed by ten healthy adult subjects without any past history of orthopedic, neurological or muscloskeletal diseases. The mean ( \pm standard deviation) age $(22.5 \pm 2.6$ years $)$, height $(169.1 \pm 6.7 \mathrm{~cm})$ and weight $(57.9 \pm$ $7.4 \mathrm{~kg}$ ) were obtained for the subjects. Prior to participation, all subjects were given a verbal explanation of the experiments, and informed consent was obtained from them.

\section{Materials}

The experimental apparatus consisted of a force plate (Kistler, 9286A, $600 \times 400 \times 35 \mathrm{~mm}$ ), a threedimensional motion analysis system (DKH, FrameDIAS3D) and an electromyograph (Nihon Kohden, Multi Telemeter). The data from each apparatus was synchronized and collected into a personal computer after analog-digital conversion.

The force plate was used to trace COP during the tiptoe movement tasks. The sampling frequency was set at $1,000 \mathrm{~Hz}$. The distance of posterior COP displacement was recorded on a force platform to observe the anticipatory response.

Displacement data of body positions were measured using a three-dimensional motion analysis system. Reflective markers were placed at the following seven sites on the subjects' left side: top of the head, acromion of the shoulder, the greater trochanter of the femur, the knee joint, lateral malleolus of the fibula, the heel and the fifth metatarsal bone. Three CCD cameras were used to record the markers at a sampling frequency of 50 $\mathrm{Hz}$. The cameras were set 60 degrees apart on the left side of the subject. Three values were calculated by this system: the maximal vertical displacement and velocity of the heel and the maximal vertical displacement of the acromion during tiptoe movement task.

The surface electromyogram (EMG) activities were recorded simultaneously at the following muscles on the subjects' right side: the gastrocnemius (Gas), the tibialis anterior (TA), the biceps femoris (BF), the rectus femoris (RF), the erector spinae (ES) and the rectus abdominus (RA). The skin surface was abraded gently to reduce skin resistance to less than $10 \mathrm{k} \Omega$ using a skin polish cream. A pair of disposable surface electrodes was placed over the bellies of each muscle, $3 \mathrm{~cm}$ apart and parallel to the muscle fibers. The sampling time was set at $1,000 \mathrm{~Hz}$ and the EMG signals were band-pass filtered from 20 to $500 \mathrm{~Hz}$, incorporating a $60 \mathrm{~Hz}$ HAM filter.

\section{Procedures}

The subjects were asked to stand in a static 
position with both heels lightly touching on a force platform with their arms crossed on the chest. While standing still, the subjects were asked to look at a target point located $3 \mathrm{~m}$ in front of them and 1.5 $\mathrm{m}$ above the floor. Then, the subjects started the tiptoe movement as quickly as possible after an auditory stimulus. The tiptoe movement in this study involved two tasks: in the single task, subjects were required to move from the static standing position to the tiptoe position then return to the static standing position, all in a single movement; in the repetitive task, subjects were required to repeat the single movement task three times in succession. Prior to the actual measurement, the subjects were given sufficient practice to be able to achieve the same level in the two tasks for the maximal vertical displacement of heel. By comparing the body marker positions in the two tiptoe tasks, the initial movements could be confirmed as being identical. Furthermore, prior to the onset of tiptoe movement in both tasks, it was confirmed that the COP position in static standing was located at approximately $40 \%$ of foot length from the heel ${ }^{18)}$.

\section{Data analysis}

The data from each apparatus was saved simultaneously. From the data, the initial movements in the two tiptoe tasks were first verified to be identical. Then, to examine the action of APA responses, the EMG changes and the distance of the COP displacement were compared between the single and repetitive tasks. Finally, we examined that how the APA signals were changed in the two different tiptoe tasks initiated from same movement.

The onset of muscle activity was defined as the time from when the muscle activity exceeded 2 standard deviations of the activation level of static standing for longer than 30 milliseconds. As a focal muscle of tiptoe movement, the onset of the Gas muscle activity was set as time zero $\left(\mathrm{T}_{0}\right)$, and any muscle activities preceding the onset of Gas were regarded as anticipatory responses. Also, the time period from the onset of any muscle activities to $T_{0}$ was defined as the APA phase. As the index of magnitude of muscle activity in the APA phase, the integrated EMG (IEMG) was determined by the sum of the EMG amplitude ( $\mathrm{mV}$ ) during the APA phase (msec). According to a study by Aruin et al. ${ }^{9}$ ), in order to minimize the effects of the variability of the inter-electrode distance or the electrical resistance of the skin among subjects, the normalized IEMG was calculated using the ratio of each trial's IEMG to the maximal IEMG in 20 trials of his tiptoe movement. Furthermore, IEMG per unit time (IEMG/sec) was also calculated since the duration of muscle activity was different in each muscle as well as in each subject.

For statistical analysis, a paired t-test was used to analyze differences in the average values and a $P$ value less than 0.05 was considered statistically significant.

\section{RESULTS}

\section{Identification of initial movement in two tiptoe tasks}

To confirm whether the initial movement in the two tiptoe tasks was identical, three parameters that occur during initial tiptoe movement were compared between the two tasks: the maximal vertical displacement of the heel, that of the acromion, and the maximal velocity. The results are shown in Table 1. As no significant differences were found between the two tiptoe tasks, the initial movements were considered identical.

\section{Onset of muscle activity}

Figure 1 shows a representative example of an EMG in the two types of tiptoe task. The onset of Gas muscle activity was set as $\mathrm{T}_{0}$ as an agonist of tiptoe movement. TA and RF, which are ventral muscles in the body, both showed anticipatory muscle activity earlier than $T_{0}$. As it was difficult to determine the accurate onset of muscle activity or inhibition in RA, BF and ES, only TA and RF were used in the comparison. The onset of TA in nine of ten subjects occurred earlier in the repetitive tiptoe task than in the single task. Similarly, the onset of $\mathrm{RF}$ in all subjects occurred earlier in the repetitive tiptoe task (Table 2).

\section{Magnitude of muscle activity}

Since TA and RF showed significant differences between the single and repetitive tasks at the onset of muscle activity, the normalized IEMG results were compared between the two tiptoe tasks as an index of the magnitude of muscle activity. The normalized IEMG showed no significant difference between the two tiptoe tasks (Table 2). Furthermore, the magnitude of these muscles activity was divided by unit time (IEMG/sec). The 
Table 1. Comparison of the initiation movement between the two tiptoe tasks

\begin{tabular}{lccc}
\hline Parameters & Single task & Repetitive task & p value \\
\hline Hight of heel $(\mathrm{cm})$ & $12.4 \pm 1.1$ & $12.2 \pm 1.1$ & 0.65 \\
Velocity of heel $(\mathrm{cm} / \mathrm{s})$ & $59.8 \pm 9.8$ & $60.9 \pm 8.0$ & 0.86 \\
Hight of shoulder $(\mathrm{cm})$ & $10.5 \pm 1.6$ & $10.7 \pm 1.6$ & 0.57 \\
\hline
\end{tabular}

(Mean \pm S. D.)

There were no significant differences in each parameter between the single and repetitive tiptoe tasks. The variability of these parameters was little, thus the initial movements in the two tasks were considered identical.

results were compared between the two tiptoe tasks and a significant decrease was seen in the repetitive task compared to the single task (Table 2).

\section{Distance of posterior COP displacement}

Figure 2 represents an example of anteroposterior COP displacement during two tiptoe movements. The COP changed to the posterior preceding $\mathrm{T}_{0}$, then anterior following $\mathrm{T}_{0}$. To compare the APA response in the two tiptoe tasks, the posterior COP displacement in each task was measured. The maximal posterior COP displacement of all subjects was decreased in the repetitive task compared to the single task. The results showed a significant decrease in the repetitive task compared to the single task (Table 2).

\section{DISCUSSION}

When the single and repetitive tiptoe movement tasks, which are both initiated by identical movements, were compared to each other, the postural muscle activities and the distance of posterior COP displacement during the APA phase differed between the single and repetitive tasks. This suggests that the CNS changes the postural muscle control during the APA phase if a task becomes repetitive. Since the APA phase in the repetitive task was found to be longer than that in the single task, it implies that the CNS may provide the necessary control to stabilize posture and accomplish complex tasks. Therefore, APA may provide stability for total movements as well as for the initial parts of movements.

Change of the APA in the repetitive tiptoe movement The basic generating mechanism of APA in the tiptoe movement has been suggested ${ }^{19,20)}$ to be the same as the one observed in stepping ${ }^{17)}$ and jumping ${ }^{15)}$. When posture changes from a static standing position to tiptoeing, Gas as a plantar flexor muscle is inhibited, and TA as a dorsiflexor muscle becomes activated. This causes foot pressure to decrease in the front part of foot and increase in the rear part, temporarily displacing the COP posteriorly. This posterior COP displacement causes a positional gap between the COP and the center of mass (COM) in the anterior-posterior plane, generating an anterior moment, which then causes the heel to rise ${ }^{21)}$. Although the inhibition of Gas was not shown specifically in this study, APA might generate and lead to the tiptoe movement by the same mechanism as reported in other studies (Figs. 1 and 2). However, APA changed according to whether the tiptoe movement was a single or repetitive task. For instance, TA and RF were activated prior to onset of Gas, a primary agonist of the tiptoe movement. In addition, TA and RF in the repetitive tiptoe task were activated significantly earlier than in the single task. Since the repetitive movement task showed a prolonged APA phase, it is possible that the CNS may make different APA programs for single and repetitive tasks, despite both tasks having the same initial movement. Therefore, APA is involved in more than the generation of the initial movement. Furthermore, the APA responses in the repetitive task showed some decrease of IEMG per unit time and shorter posterior COP displacement. These results also support the possibility that different APA programs might be set for each task. Since both tiptoe tasks were initiated from an identical movement, one of the reasons why APA changes according to the task might be an effect of the complexity that repeated 
single
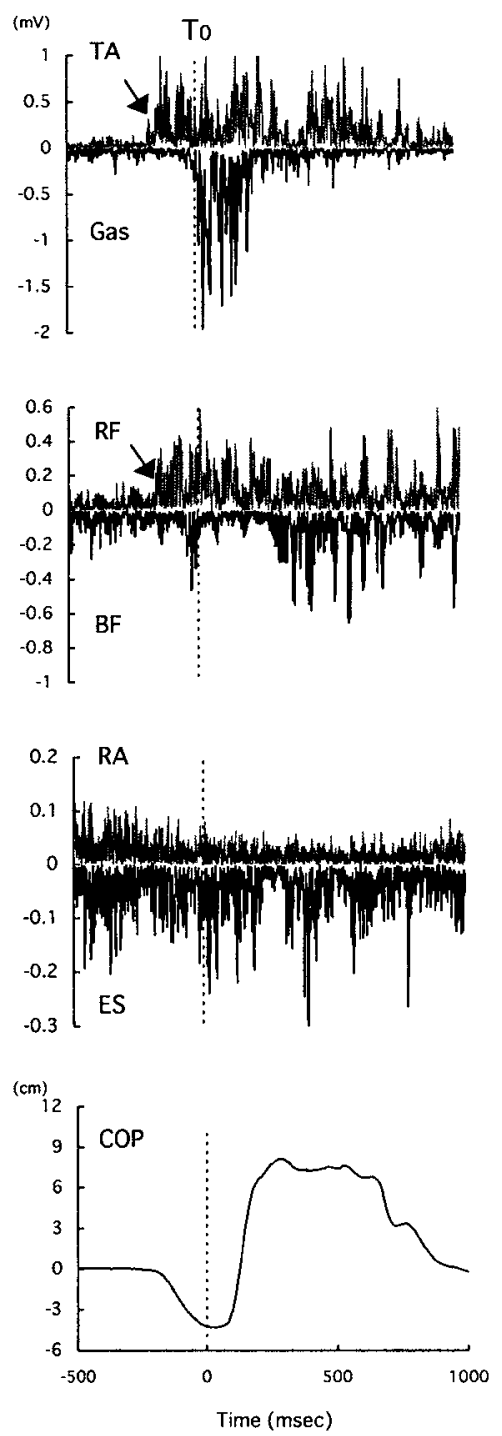

repetitive
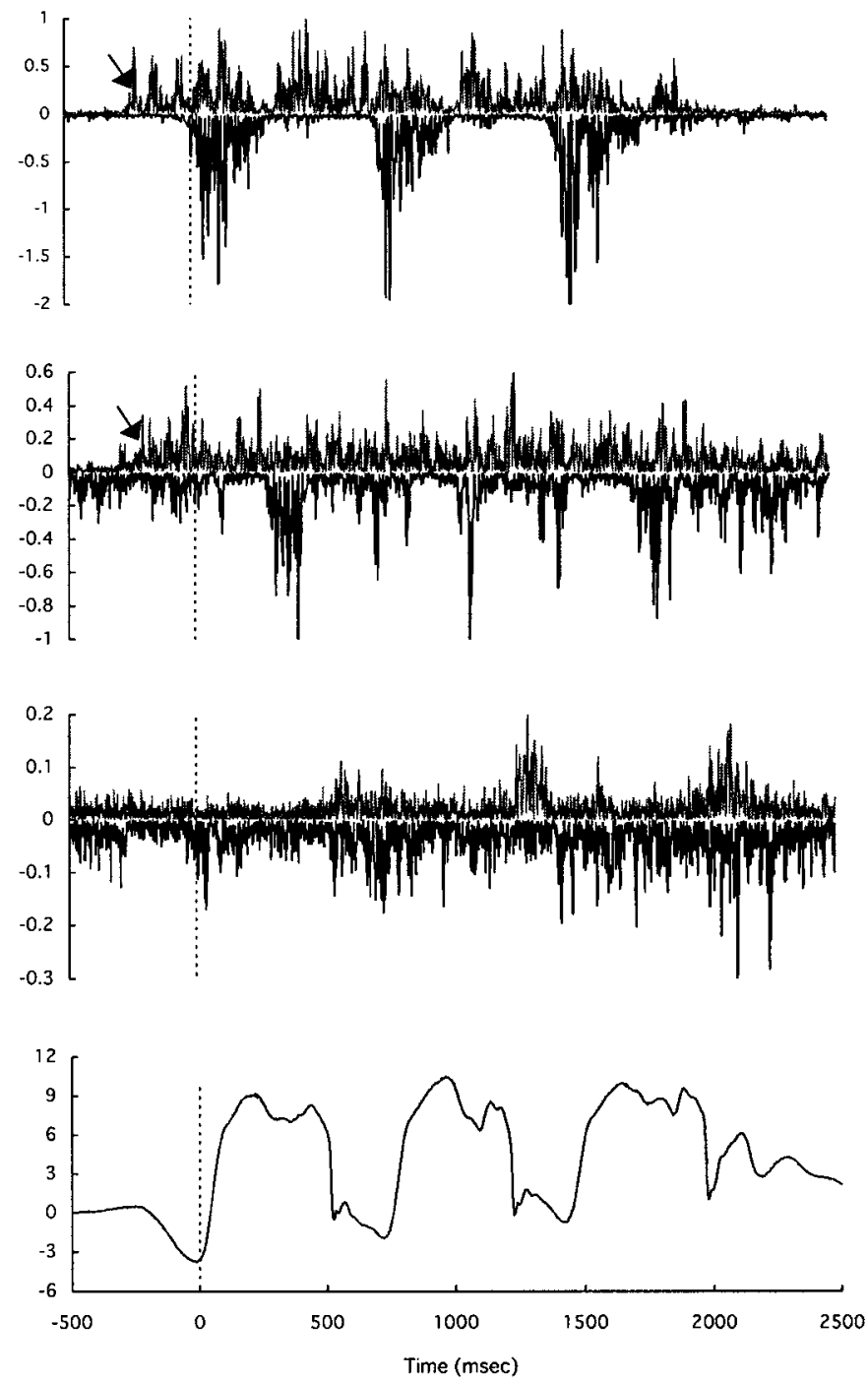

Fig. 1. EMG and COP traces from one subject during the two tiptoe tasks. Traces are aligned to the onset of the gastrocnemius, indicated by the dashed vertical line $\left(\mathrm{T}_{0}\right)$. The amplitude of the dorsal muscle activities (gastrocnemius, biceps femoris and erector spinae) is shown as a negative value. Anticipatory muscle responses in TA and RF preceding the onset of Gas were confirmed in both tiptoe tasks (shown by arrowhead). Simultaneously, COP posterior displacement started. The muscle responses were earlier during the repetitive task than in the single task.

movement tasks require. In the studies of Lipshits et al. ${ }^{22)}$ and Pellec et al. ${ }^{15)}$, APA and the postural muscle activities changed according to the complexity of the task.

Role of APA on the movement task associated with changing $B O S$

Compared to the single tiptoe task, APA responses in the repetitive task showed a significantly prolonged APA phase and a significant decrease of IEMG per unit time. However, when the magnitude of muscle activity in the whole APA phase was examined, the total magnitude of it did not change in either task, as shown in the comparison of the normalized IEMG (Table 2). These suggest that the CNS does not change the total magnitude of muscle activity until the actual initial movement is executed, and ensures 
Table 2. Comparison of the APA responses between the two tiptoe tasks

\begin{tabular}{llrrr}
\hline Parameters & & \multicolumn{1}{c}{ Single task } & Repetitive task & p value \\
\hline Onset $(\mathrm{ms})$ & TA & $262.3 \pm 79.3$ & $293.2 \pm 74.0$ & $<0.05$ \\
& RF & $236.8 \pm 94.7$ & $265.8 \pm 75.0$ & $<0.05$ \\
Normalized IEMG & TA & $0.72 \pm 0.09$ & $0.68 \pm 0.12$ & 0.53 \\
& RF & $0.68 \pm 0.16$ & $0.62 \pm 0.13$ & 0.30 \\
Normalized IEMG/sec & TA & $3.29 \pm 0.79$ & $2.63 \pm 0.80$ & $<0.05$ \\
& RF & $2.82 \pm 1.36$ & $2.06 \pm 0.92$ & $<0.05$ \\
COP displacement $(\mathrm{cm})$ & & $5.2 \pm 1.30$ & $5.7 \pm 1.10$ & $<0.01$ \\
\hline
\end{tabular}

(Mean \pm S. D.)

Onset: the lead time of the onset of each muscle for Gas $\left(\mathrm{T}_{0}\right)$. IEMG: integrated EMG during the APA phase. Each IEMG was normalized by maximal IEMG in all trials for each subject. IEMG/sec: IEMG per unit time. COP displacement: maximal posterior COP displacement.

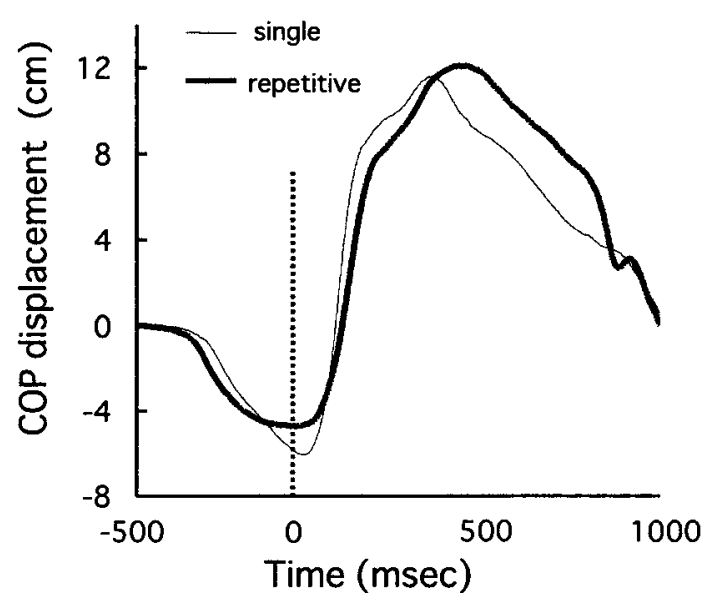

Fig. 2. Representative example of COP displacement. Vertical line indicates onset of Gas $\left(\mathrm{T}_{0}\right)$. The COP changed to posterior preceding $\mathrm{T}_{0}$ in the both tasks. The peak of the posterior COP displacement occurred almost at the same time as $\mathrm{T}_{0}$.

stable movement by modulation of time and amplitude, both of which are factors in determining the magnitude of muscle activity. This suggestion is also supported by the comparison of COP displacement (Table 2), in which the distance of posterior COP displacement was decreased in the repetitive task, which required more stability than the single task. In a movement task associated with changing BOS, therefore, it can be thought that the CNS not only provides the driving force to initiate movement, but also the appropriate control to efficiently execute the planned movement. Some studies $^{23-25)}$ on APA have reported that the CNS works to elicit the biomechanical properties of programmed movement in advance, and also controls postural instability occurring in the initial and final movements.

Compared to healthy subjects, patients with the CNS problems and the elderly have been reported to show weak $\mathrm{APA}^{26,27)}$. Also, in their rehabilitation, it has been reported that postural muscle activities were of great importance for accomplishing stable movements ${ }^{28)}$. Therefore, any rehabilitation should include treatment plans aimed at APA, and perform repetitive or repeated movements since our daily activities are composed of many repetitive movements.

\section{REFERENCES}

1) Belen'kii VE, Gurfinkel' VS, Pal'tsev EI: Control Elements of voluntary movements. Biofizika, 1967, 12: $135-141$.

2) Hugon M, Massion J, Wiesendanger M: Anticipatory postural changes induced by active unloading and comparison with passive unloading in man. Pflugers Arch, 1982, 393: 292-296.

3) Bouisset M, Zattara M: A sequence of postural movements precedes voluntary movement. Neurosci Lett, 1981, 22: 263-270.

4) Winstein CJ, Horak FB, Fisher BE: Influence of central set on anticipatory and triggered grip-force adjustments. Exp Brain Res, 2000, 130: 298-308.

5) Bonnetblanc F, Martin O, Teasdale N: Pointing to a target from an upright standing position: anticipatory postural adjustments are modulated by the size of the target in humans. Neurosci Lett, 2004, 358: 181-184.

6) Lepers R, Breniere Y: The role of anticipatory postural adjustments and gravity in gait initiation. Exp Brain Res, 1995, 107: 118-124. 
7) Pellec A, Maton B: Anticipatory postural adjustments are associated with single vertical jump and their timing is predictive of jump amplitude. Exp Brain Res, 1999, 129: 551-558.

8) Latash ML, Aruin AS, Neyman I, et al.: Anticipatory postural adjustments during self inflicted and predictable perturbations in Parkinson's disease. J Neurol Neurosurg Psychiatry, 1995, 58: 326-334.

9) Aruin AS, Shiratori T, Latash ML: The role of action in postural preparation for loading and unloading in standing subjects. Exp Brain Res, 2001, 138: 458-466.

10) Cordo P, Nashner L: Properties of postural adjustments associated with rapid arm movements. J Neurophysiol, 1982, 47: 287-302.

11) Bent L, Potvin J, Brooke JD, et al.: Medio-lateral balance adjustments preceding reflexive limb withdrawal are modified by postural demands. Brain Res, 2001, 914: 100-105.

12) Zettel JC, McIlroy WE, Maki BE: Environmental constraints on foot trajectory reveal the capacity for modulation of anticipatory postural adjustments during rapid triggered stepping reactions. Exp Brain Res, 2002, 146: 38-47.

13) Massion J: Movement, posture and equilibrium: interaction and coordination. Prog Neurobiol, 1992, 38: 35-56.

14) Breniere Y, Do MC, Sanchez J: A biomechanical study of the gait initiation process. J Biophys Med Nucl, 1981, 5: 197-206.

15) Pellec A, Maton B: Anticipatory postural adjustments depend on final equilibrium and task complexity in vertical high jump movements. J Electromyogr Kinesiol, 2000, 10: 171-178.

16) Martin O, Teasdale N, Simoneau M, et al.: Pointing to a target from an upright position in human: tuning of postural responses when there is target uncertainty. Neurosci Lett, 2000, 281: 53-56.

17) Breniere $Y$, Do M: Control of gait initiation. J Mot Behav, 1991, 23: 235-240.

18) Fujiwara K, Ikegami H: A study on the relationship between the position of the center of foot pressure and the steadiness of standing posture. Jap J Phys Educ, 1981, 26: 137-147.

19) Nardone A, Schieppati M: Postural adjustments associated with voluntary contraction of leg muscles in standing man. Exp Brain Res, 1988, 69: 469-480.

20) Yamashita N, Moritani $T$ : Anticipatory changes of soleus H-reflex amplitude during execution process for heel raise from standing position. Brain Res, 1989, 490: $148-151$.

21) Ito $\mathrm{T}$, Azuma $\mathrm{T}$, Yamashita $\mathrm{N}$ : Anticipatory control related to the upward propulsive force during the rising on tiptoe from an upright standing position. Eur J Appl Physiol, 2004, 92: 186-195.

22) Lipshits MI, Mauritz K, Popov KE: Quantitative analysis of anticipatory postural components of a complex voluntary movement. Hum Physiol, 1981, 7: 165-173.

23) Kasai T, Kawai K: Quantitative EMG analysis of anticipatory postural adjustments of voluntary contraction of leg muscles in standing man. Electroencephalogr Clin Neurophysiol, 1994, 93: 184187.

24) Mille M, Mouchnino L: Are human anticipatory postural adjustments affected by a modification of the initial position of the center of gravity? Neurosc Lett, 1998, 242: 61-64.

25) Nardonne A, Schieppati M: Postural adjustments associated with voluntary contractions of leg muscles in standing man. Exp Brain Res, 1988, 69: 469-480.

26) Garland SJ, Stevenson TJ, Ivanova T: Postural responses to unilateral arm perturbation in young, elderly, and hemiplegic subjects. Arch Phys Med Rehabil, 1997, 78: 1072-1077.

27) Traub MM, Rothwell JC, Marsden CD: Anticipatory postural reflexes in Parkinson's disease and other akinetic-rigid syndromes and in cerebellar ataxia. Brain, 1980, 103: 393-412.

28) Dickstein R, Shefi S, Marcovitz E, et al.: Anticipatory postural adjustment in selected trunk muscles in post stroke hemiparetic patients. Arch Phys Med Rehabil, 2004, 85: 261-267. 